\title{
Band structure and optical transitions in atomic layers of hexagonal gallium chalcogenides
}

\author{
V. Zólyomi, N. D. Drummond, and V. I. Fal'ko \\ Physics Department, Lancaster University, Lancaster LAI 4YB, United Kingdom
}

(Received 20 February 2013; revised manuscript received 15 April 2013; published 2 May 2013)

\begin{abstract}
We report density-functional-theory calculations of the electronic band structures and optical absorption spectra of two-dimensional crystals of $\mathrm{Ga}_{2} X_{2}(X=\mathrm{S}$, Se, and Te). Our calculations show that all three two-dimensional materials are dynamically stable indirect-band-gap semiconductors with a sombrero dispersion of holes near the top of the valence band. We predict the existence of Lifshitz transitions-changes in the Fermi-surface topology of hole-doped $\mathrm{Ga}_{2} X_{2}$-at hole concentrations $n_{S}=7.96 \times 10^{13} \mathrm{~cm}^{-2}, n_{S e}=6.13 \times 10^{13} \mathrm{~cm}^{-2}$, and $n_{T e}=3.54 \times 10^{13} \mathrm{~cm}^{-2}$.
\end{abstract}

DOI: 10.1103/PhysRevB.87.195403

PACS number(s): 73.61.Ga, 71.15.Mb, 73.20.-r, 78.66.Hf

\section{INTRODUCTION}

Two-dimensional semiconductors have been studied intensively in the past few decades as researchers have tried to find materials to complement or even replace silicon in electronics. A recent trend in the hunt for new two-dimensional systems consists of the isolation and study of atomically thin sheets of layered materials. Honeycomb carbon allotropes have been widely investigated for this purpose following the discovery of graphene, ${ }^{1,2}$ hexagonal boron nitride is another example., With increasing chemical unit cell complexity, hexagonal transition metal dichalcogenides have been exfoliated into atomically thin films using mechanical transfer and liquidphase sonication; ${ }^{5-9}$ many of these materials are semiconductors in both bulk and monolayer phases, making them ideal for use in single-sheet nanoscale optoelectronics. ${ }^{6,8,10,11}$ In this work we explore the next generation in the family of two-dimensional atomic crystals: stoichiometric layers of gallium chalcogenides $\left(\mathrm{Ga}_{2} X_{2}\right)$.

In the bulk form, $\mathrm{GaS}$ and $\mathrm{GaSe}$ are indirect-gap semiconductors, ${ }^{12}$ with the latter being well known for its nonlinear optical properties, ${ }^{13}$ while GaTe is a direct-gap semiconductor used for electrothermal threshold switching. ${ }^{14}$ All three materials exhibit the same structure: they are layered compounds of $\mathrm{Ga}_{2} \mathrm{X}_{2}$ stoichiometry in which each layer consists of two AA-stacked hexagonal sublayers of gallium atoms sandwiched between two hexagonal sublayers of chalcogen atoms $(X)$, as illustrated in Fig. 1. In a bulk material, these layers are bound in a three-dimensional structure by van der Waals interactions.

In this work we discuss the optical and electronic properties of single-layer crystals of $\mathrm{Ga}_{2} \mathrm{~S}_{2}, \mathrm{Ga}_{2} \mathrm{Se}_{2}$, and $\mathrm{Ga}_{2} \mathrm{Te}_{2}$. We present the electronic band structures (see Fig. 2) and optical absorption spectra for all three materials. In each case we find that the band gap is indirect, with the valence-band maximum shifted from the $\Gamma$ point (an inverted sombrero dispersion) and the conduction-band minimum (CBM) located at the $M$ point in $\mathrm{Ga}_{2} \mathrm{~S}_{2}$ and $\mathrm{Ga}_{2} \mathrm{Te}_{2}$, and at the $\Gamma$ point in $\mathrm{Ga}_{2} \mathrm{Se}_{2}$. For all three materials we find an unusual, slightly anisotropic sombrero shape in the electron dispersion near the edge of the valence band. We find that hole-doped $\mathrm{Ga}_{2} X_{2}$ undergoes a Lifshitz transition ${ }^{15}$ - a change in the topology of the Fermi surface illustrated in Fig. 3-at hole concentrations $n_{S}=7.96 \times 10^{13}$ $\mathrm{cm}^{-2}, n_{S e}=6.13 \times 10^{13} \mathrm{~cm}^{-2}$, and $n_{T e}=3.54 \times 10^{13} \mathrm{~cm}^{-2}$. However, the strongest peak in the optical absorption spectra
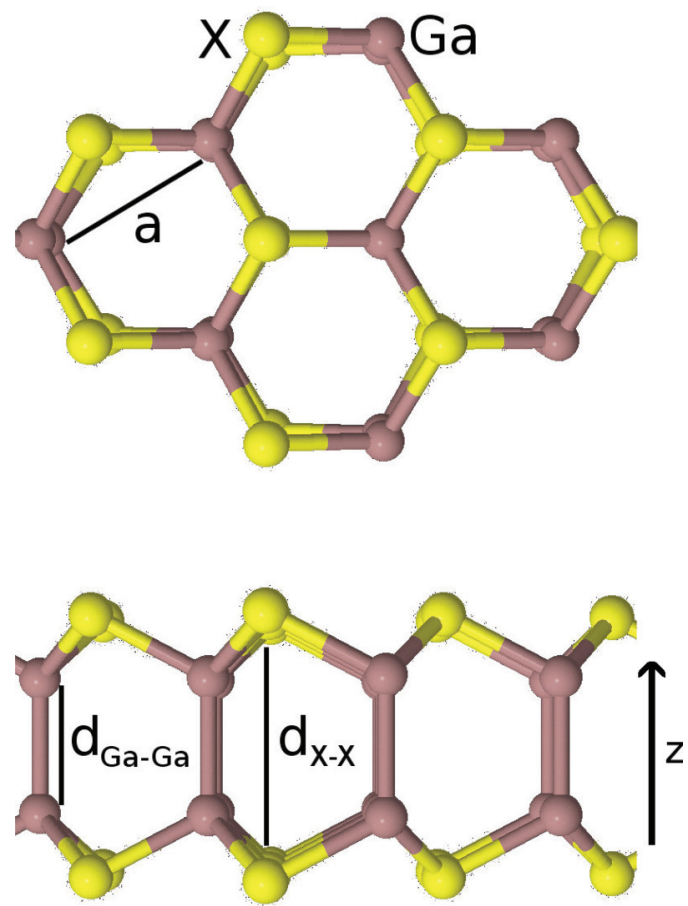

FIG. 1. (Color online) Structure of monolayer gallium chalcogenides $\mathrm{Ga}_{2} X_{2}(X=\mathrm{S}, \mathrm{Se}, \mathrm{Te})$ in top and side views.

shown in Fig. 4-dominated by a transition between two bands with states which are even with respect to $z \rightarrow-z$ symmetryis not caused by the Van Hove singularity associated with the sombrero spectra, but is provided by two, near-parallel branches in the dispersion of the conduction bands near the Brillouin-zone corners $K$ and $K^{\prime}$.

TABLE I. Structural parameters (as defined in Fig. 1) of singlelayer $\mathrm{Ga}_{2} \mathrm{X}_{2}$ (in $\AA$ ) from the DFT calculations.

\begin{tabular}{lcccccc}
\hline \hline$X$ & $a^{\mathrm{LDA}}$ & $d_{\mathrm{Ga}-\mathrm{Ga}}^{\mathrm{LDA}}$ & $d_{X-X}^{\mathrm{LDA}}$ & $a^{\mathrm{PBE}}$ & $d_{\mathrm{Ga}-\mathrm{Ga}}^{\mathrm{PBE}}$ & $d_{X-X}^{\mathrm{PBE}}$ \\
\hline $\mathrm{S}$ & 3.53 & 2.41 & 4.57 & 3.63 & 2.47 & 4.64 \\
$\mathrm{Se}$ & 3.71 & 2.40 & 4.73 & 3.82 & 2.47 & 4.82 \\
$\mathrm{Te}$ & 4.01 & 2.40 & 4.92 & 4.14 & 2.47 & 5.02 \\
\hline \hline
\end{tabular}



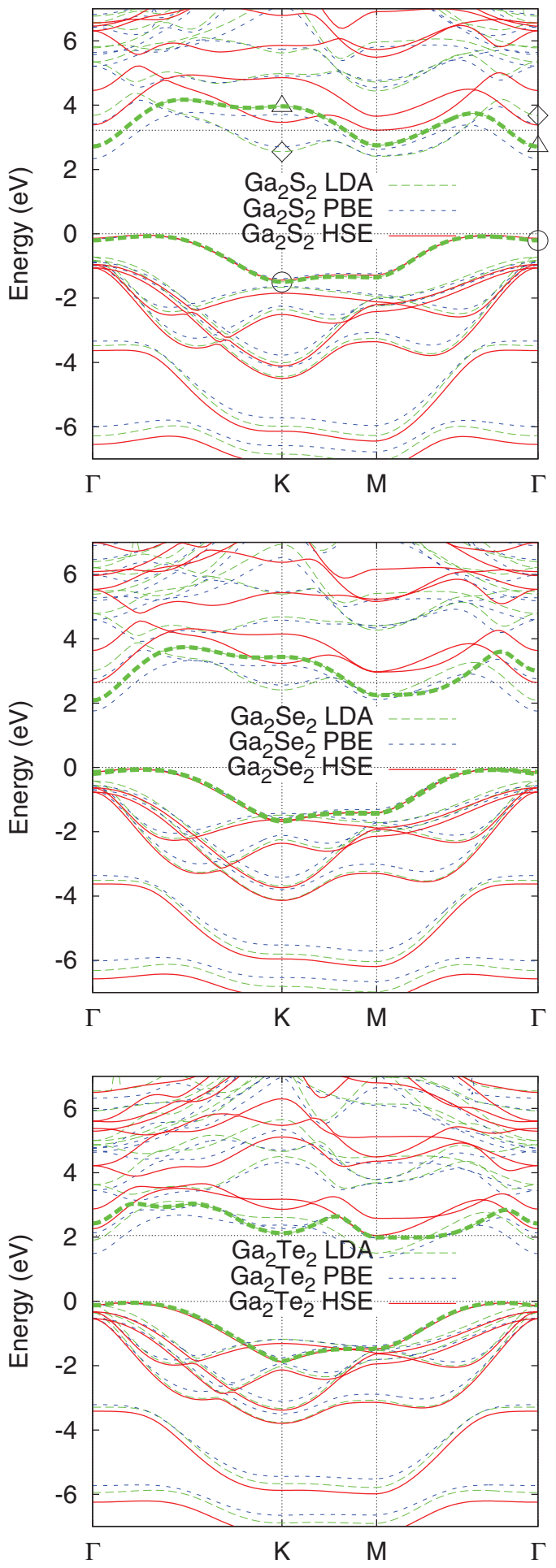

FIG. 2. (Color online) HSE06 band structures (solid red lines) for $\mathrm{Ga}_{2} \mathrm{~S}_{2}$ (top), $\mathrm{Ga}_{2} \mathrm{Se}_{2}$ (middle), and $\mathrm{Ga}_{2} \mathrm{Te}_{2}$ (bottom). The zero of energy is taken to be the Fermi level and the bottom of the conduction band is marked with a horizontal line. For comparison, the semilocal band structures are also shown. In the case of the LDA the two symmetric bands that give rise to the main feature in the optical absorption spectrum are marked in bold.

\section{RESULTS AND DISCUSSION}

The band-structure analysis of single-layer $\mathrm{Ga}_{2} X_{2}$ leading to the above conclusions was performed using density-

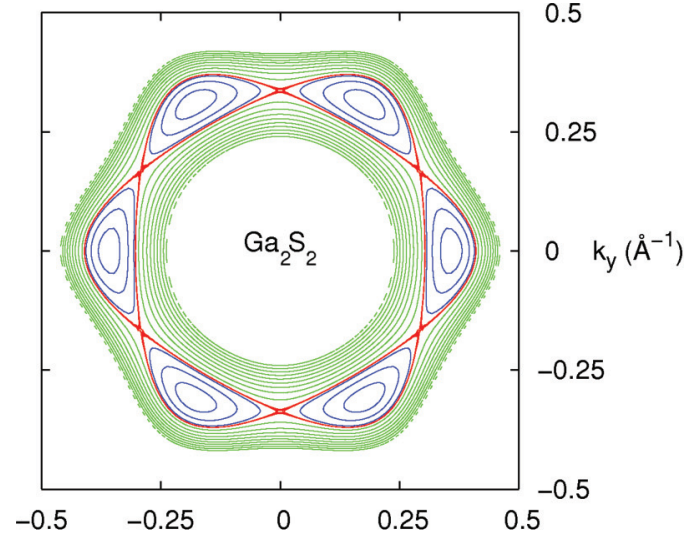

$k_{x}\left(\AA^{-1}\right)$

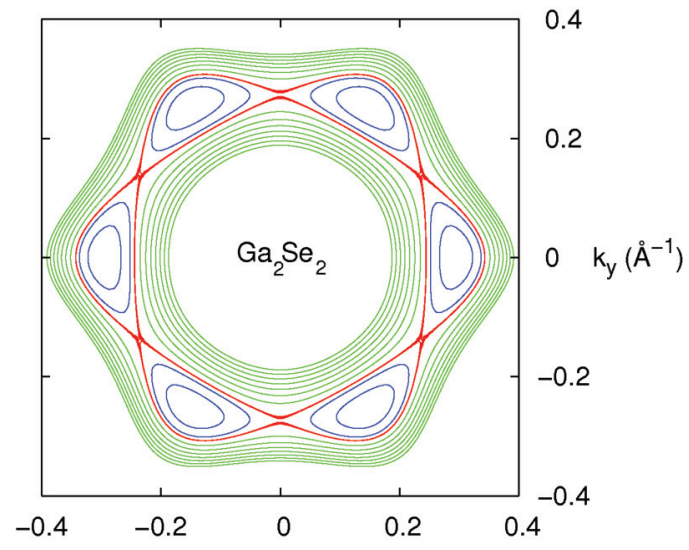

$k_{x}\left(\AA^{-1}\right)$

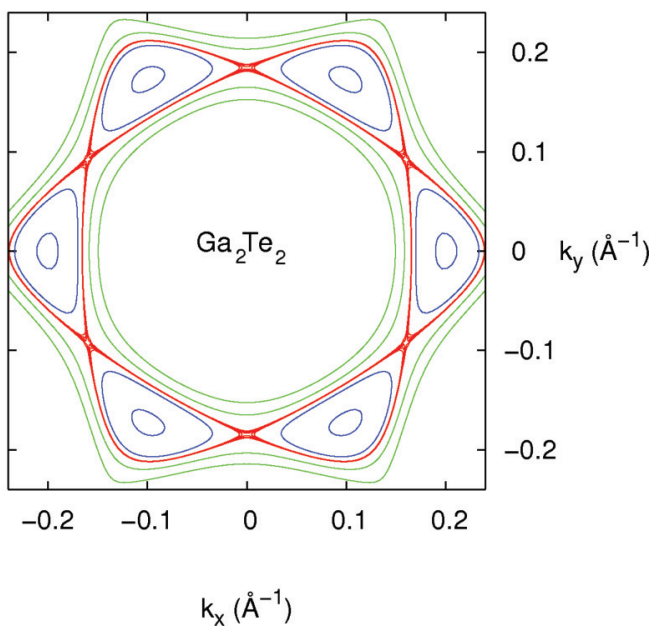

FIG. 3. (Color online) Energy contours of $\mathrm{Ga} X$ in the valence band using the fitted formula of Eq. (1). The separation of contours is $2 \mathrm{meV}$ in all figures and the contour corresponding to the energy of the saddle point along the $\Gamma-M$ line is highlighted.

functional theory (DFT) as implemented in the CASTEP ${ }^{16}$ and VASP $^{17}$ plane-wave-basis codes. Tests have shown that the two codes yield nearly indistinguishable results for the materials studied here. To calculate the geometries, phonon dispersions, and optical absorption spectra, we used semilocal exchangecorrelation functionals: the local density approximation (LDA) 

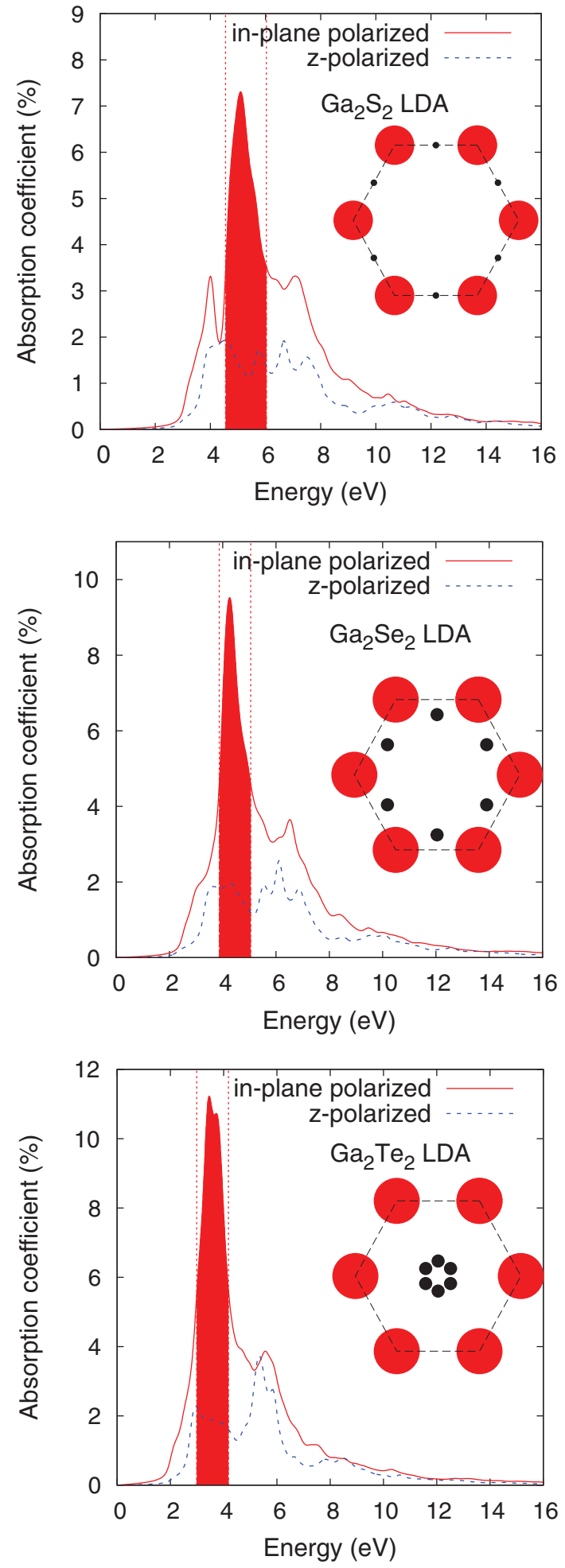

FIG. 4. (Color online) Absorption coefficient (the imaginary part of the dielectric function $\varepsilon$ ) of various $\mathrm{Ga}_{2} \mathrm{X}_{2}$ two-dimensional crystals normalized to absolute units after it was compared to $\operatorname{Im}(\varepsilon)$ evaluated for graphene in the range $0.8-1.5 \mathrm{eV}$, where monolayer graphene absorbs $2.3 \%$ of light (see text for details). Vertical lines mark the full width at half maximum for the main peak. The insets illustrate the relevant part of $\mathbf{k}$-space for the transitions between bands marked by circle and diamond in Fig. 2 which give rise to the main peak (light red circle) and the low-energy shoulder (black circle).
TABLE II. Coefficients $E_{2 i}$ (in units of $\mathrm{eV} \AA^{2 i}$ ) for the inverted sombrero dispersion near the VBM in Eq. (1). The root mean squares of the residuals in $\mathrm{Ga}_{2} \mathrm{~S}_{2}, \mathrm{Ga}_{2} \mathrm{Se}_{2}$, and $\mathrm{Ga}_{2} \mathrm{Te}_{2}$ were found to be 0.48 , 0.68 , and $0.67 \mathrm{meV}$ respectively. The fitting parameters were obtained by fitting the formula of Eq. (1) to the DFT data within the ranges of $0.28<|\mathbf{k}|<0.42,0.22<|\mathbf{k}|<0.36$, and $0.12<|\mathbf{k}|<0.26$ in $\mathrm{Ga}_{2} \mathrm{~S}_{2}, \mathrm{Ga}_{2} \mathrm{Se}_{2}$, and $\mathrm{Ga}_{2} \mathrm{Te}_{2}$, respectively (with $\mathbf{k}$ in units of $\AA^{-1}$ ). The zero of energy is set to the valence-band maximum. The last column shows the critical hole concentration $\left(n_{X}\right.$, in units of $\left.\mathrm{cm}^{-2}\right)$ where the Lifshitz transition takes place (see text).

\begin{tabular}{lcccccc}
\hline \hline $\mathrm{X}$ & $\mathrm{E}_{0}$ & $\mathrm{E}_{2}$ & $\mathrm{E}_{4}$ & $\mathrm{E}_{6}$ & $\mathrm{E}_{6}^{\prime}$ & $n_{X}$ \\
\hline $\mathrm{S}$ & -0.086 & 1.38 & -6.8 & 5.6 & 1.634 & $7.96 \times 10^{13}$ \\
$\mathrm{Se}$ & -0.059 & 1.49 & -11.4 & 17 & 4.49 & $6.13 \times 10^{13}$ \\
$\mathrm{Te}$ & -0.044 & 2.50 & -47 & 240 & 30.1 & $3.54 \times 10^{13}$ \\
\hline \hline
\end{tabular}

and the Perdew-Burke-Ernzerhof ${ }^{18}$ (PBE) functionals. The screened Heyd-Scuseria-Ernzerhof 06 (HSE06) functional ${ }^{19}$ was used to obtain the electronic band structures to compensate at least partially for the underestimation of the band gap by semilocal functionals. The plane-wave cutoff energy was $600 \mathrm{eV}$. During relaxations a $12 \times 12$ Monkhorst-Pack k-point grid was used, while band structures were obtained with a $24 \times 24$ grid. The optical absorption spectra were obtained with a very dense grid of $95 \times 95 \mathbf{k}$ points. The artificial out-of-plane periodicity of the monolayer was set to $20 \AA$. Phonon dispersions were calculated in $\operatorname{CASTEP}^{20}$ using the method of finite displacements in a $4 \times 4$ supercell, with $11 \times 11$ k points, a $408 \mathrm{eV}$ plane-wave cutoff, and an artificial out-of-plane periodicity of $15.88 \AA$.

Full geometry optimization was performed using both the LDA and PBE functionals. Table I shows that the lattice constants increase with the atomic number of the chalcogen atom, while the Ga-Ga bond lengths hardly change. The bond lengths obtained with the PBE functional are systematically larger than those optimized with the LDA, as expected. ${ }^{21}$

The calculated electronic band structures are summarized in Fig. 2. All three materials are indirect-gap semiconductors, primarily due to the valence-band maximum (VBM) lying somewhat off the $\Gamma$ point. It is possible to fit an inverted sombrero polynomial to the valence-band dispersions $E_{V B}$ around the valence-band maximum:

$$
E_{V B}=\sum_{i=0}^{3} E_{2 i} k^{2 i}+E_{6}^{\prime} k^{6} \cos 6 \varphi,
$$

where $k$ and $\varphi$ are the polar coordinates of the wave vectors measured from the $\Gamma$ point in units of $1 / \AA, \varphi$ is measured from

TABLE III. The band gaps of $\mathrm{Ga}_{2} X_{2}$ according to the HSE06 functional $\left(\Delta_{H S E}\right)$ and the spin-orbit splitting in the LDA band structure at the $K$ point in the highest valence $\left(\Delta E_{S O}^{v, \mathrm{~K}}\right)$ and lowest conduction $\left(\Delta E_{S O}^{c, K}\right)$ bands.

\begin{tabular}{lcrr}
\hline \hline$X$ & $\Delta_{H S E}$ & $\left|\Delta E_{S O}^{v, \mathrm{~K}}\right|$ & $\left|\Delta E_{S O}^{c, K}\right|$ \\
\hline $\mathrm{S}$ & $3.28 \mathrm{eV}$ & $19 \mathrm{meV}$ & $21 \mathrm{meV}$ \\
$\mathrm{Se}$ & $2.69 \mathrm{eV}$ & $2 \mathrm{meV}$ & $36 \mathrm{meV}$ \\
$\mathrm{Te}$ & $2.10 \mathrm{eV}$ & $14 \mathrm{meV}$ & $102 \mathrm{meV}$ \\
\hline \hline
\end{tabular}


TABLE IV. The effective masses $\left(m^{*} / m_{e}\right)$ of $\mathrm{Ga}_{2} X_{2}$ at the highsymmetry points in the conduction band according to the HSE06 functional (in units of electron mass).

\begin{tabular}{lcccc}
\hline \hline$X$ & $\Gamma^{c}$ & $K^{c}$ & $M^{c} \rightarrow \Gamma$ & $M^{c} \rightarrow \mathrm{K}$ \\
\hline $\mathrm{S}$ & 0.23 & 0.62 & 1.52 & 0.29 \\
$\mathrm{Se}$ & 0.17 & 0.56 & 3.03 & 0.23 \\
$\mathrm{Te}$ & 0.14 & 0.47 & 0.43 & 0.19 \\
\hline \hline
\end{tabular}

the $\Gamma-K$ line, and the energy is in units of eV. The coefficients are listed in Table II. This fit should provide a good starting point for a simple analytical model of the valence band in these materials. Note, however, that this fit is designed to describe the immediate vicinity of the VBM and the saddle point, and it no longer describes the $\Gamma$ point correctly, overestimating the $\Gamma$ point valence band energy by 52,70 , and $32 \mathrm{meV}$ in $\mathrm{Ga}_{2} \mathrm{~S}_{2}$, $\mathrm{Ga}_{2} \mathrm{Se}_{2}$, and $\mathrm{Ga}_{2} \mathrm{Te}_{2}$, respectively.

Further analysis of the valence band reveals a saddle point along the $\Gamma-M$ line, illustrated in Fig. 3. This saddle point gives rise to a Van Hove singularity in the density of states quite close to the Fermi level, which is found to be at the valence-band edge. Due to the presence of these saddle points, hole doping causes $\mathrm{Ga}_{2} X_{2}$ to undergo a Lifshitz transition when the hole concentration reaches the critical value where all states are depleted above the energy of the saddle point, since this leads to a change in the topology of the Fermi surface. The carrier density where the Lifshitz transition takes place in each material is listed in the last column of Table II and was obtained by integrating the DFT density of states from the saddle point to the valence-band edge.

We find that the conduction-band minimum (CBM) is at the $M$ point in $\mathrm{Ga}_{2} \mathrm{~S}_{2}$ and $\mathrm{Ga}_{2} \mathrm{Te}_{2}$, and at the $\Gamma$ point in $\mathrm{Ga}_{2} \mathrm{Se}_{2}$. The HSE06 band gaps of $\mathrm{Ga}_{2} X_{2}$ are summarized in Table III. The HSE06 band gap is expected to underestimate the quasiparticle band gap by no more than $10 \%,{ }^{22}$ and is known to be applicable to two-dimensional materials as well. ${ }^{23}$ Our finding that the gap is indirect is in agreement with a recent DFT result obtained for single-layer $\mathrm{Ga}_{2} \mathrm{Se}_{2},{ }^{24}$ although density-functional tight-binding calculations ${ }^{25}$ disagree with our results for $\mathrm{Ga}_{2} \mathrm{~S}_{2}$. The effective masses at the highsymmetry points in the conduction band are summarized in Table IV. The effective mass is isotropic at $\Gamma$ and $K$, but not at the $M$ point. The semilocal band structures are also plotted in Fig. 2 for comparison. The LDA and PBE functionals give very similar results to the HSE06 functional up to the Fermi level, but above that significant discrepancies arise. This is most notable in the case of $\mathrm{Ga}_{2} \mathrm{~S}_{2}$, where the position of the
CBM is ambiguous: the LDA predicts that the CBM is at the $M$ point, in agreement with HSE06, while the PBE functional puts it at the $\Gamma$ point. In $\mathrm{Ga}_{2} \mathrm{Se}_{2}$ and $\mathrm{Ga}_{2} \mathrm{Te}_{2}$ both the LDA and the PBE functionals predict that the CBM is at the same place as in the HSE06 calculation.

Table $\mathrm{V}$ shows an orbital composition analysis of the LDA bands of $\mathrm{Ga}_{2} \mathrm{~S}_{2}$ around the Fermi level. We have found that these bands are dominated by $s$ - and $p$-type orbitals. Furthermore, states in each band are either odd or even with respect to $z \rightarrow-z$ symmetry (this information is obtained from the complex phases of the orbital decomposition in VASP). Therefore, the interband absorption selection rules require that photons polarized in the plane of the two-dimensional crystal are absorbed by transitions between bands with the same $z \rightarrow-z$ symmetry of wave functions (even $\rightarrow$ even, odd $\rightarrow$ odd), and photons polarized along the $z$ axis cause transitions between bands with opposite symmetry (even $\rightarrow$ odd, odd $\rightarrow$ even). Note that in the case of $\mathrm{Ga}_{2} \mathrm{Te}_{2}$ these numbers only provide a qualitative description of whether the orbitals are of mostly $s$ or $p$ character due to the strong spin-orbit coupling.

The calculated optical absorption spectra (using semilocal DFT) are shown in Fig. 4. The intensities were normalized by using graphene as a benchmark. We calculated the absorption of graphene using the same method in the $0.8-1.5 \mathrm{eV}$ energy range where monolayer graphene absorbs $2.3 \%$ of light, and used the calculated graphene absorption intensity to obtain a scaling factor that scales the calculated intensities to the experimental value. Since in the case of $\mathrm{Ga}_{2} \mathrm{~S}_{2}$ we found disagreement between the PBE and the HSE06 regarding the location of the CBM, we restrict ourselves to the LDA in the following. Note that the LDA results are only qualitatively accurate and should only be used for a comparative study of the different $\mathrm{Ga}_{2} \mathrm{X}_{2}$ monolayers and for an order-of-magnitude estimate of the expected peak positions. A better description would require a computationally much more expensive calculation using the GW approximation and the Bethe-Salpeter equation for excitonic corrections. ${ }^{26}$ In Fig. 4 we show the energy dependence of the absorption coefficients of various stoichiometric $\mathrm{Ga}_{2} X_{2}$ monolayers. The absorption spectrum starts with a shoulder at low energies, which originates from the vicinity of the $M$ and $\Gamma$ points. In all cases the in-plane absorption is much more significant and is dominated by peaks at around 5, 4, and $3.5 \mathrm{eV}$ for $X=\mathrm{S}$, Se, and Te, respectively. Analysis of the band structure reveals that the bulk of this spectrum comes from transitions between the valence band and the $z \rightarrow-z$ even band above the conduction band around the $K$ point where the two bands are near parallel (this is the only part in the band structure where the energy difference

TABLE V. Orbital analysis of the LDA bands around the Fermi level in $\mathrm{Ga}_{2} \mathrm{~S}_{2}$ at the $\Gamma$ and $K$ points. The labels $\bigcirc, \triangle$, and $\diamond$ correspond to the bands labeled as such in Fig. 2. The data were obtained by projecting the orbitals in the plane-wave basis set of VASP onto spherical harmonics. Dominant contributions were found to originate from $s$ and $p$ type orbitals; +- subscripts refer to even $(+)$ and odd $(-)$ states with respect to $z \rightarrow-z$ reflection.

\begin{tabular}{lll}
\hline \hline Band & $\Gamma$ & \multicolumn{1}{c}{$K$} \\
\hline$\bigcirc_{+}$ & $0.014 s^{\mathrm{Ga}}+0.058 p_{z}{ }^{\mathrm{Ga}}+0.004 s^{\mathrm{S}}+0.189 p_{z}{ }^{\mathrm{S}}$ & $0.058 s^{\mathrm{Ga}}+0.169 p_{z}{ }^{\mathrm{Ga}}+0.044 p_{x} p_{y}{ }^{\mathrm{S}}$ \\
$\triangle_{-}$ & $0.142 s^{\mathrm{Ga}}+0.008 p_{z}{ }^{\mathrm{Ga}}+0.098 s^{\mathrm{S}}+0.056 p_{z} \mathrm{~S}$ & $0.190 s^{\mathrm{Ga}}+0.008 p_{z}{ }^{\mathrm{Ga}}+0.051 p_{x} p_{y}{ }^{\mathrm{S}}$ \\
$\diamond_{+}$ & $0.075 s^{\mathrm{Ga}}+0.107 p_{z}{ }^{\mathrm{Ga}}+0.082 s^{\mathrm{S}}+0.001 p_{z}{ }^{\mathrm{S}}$ & $0.030 p_{x} p_{y}{ }^{\mathrm{Ga}}+0.036 p_{x} p_{y}{ }^{\mathrm{S}}$ \\
\hline \hline
\end{tabular}



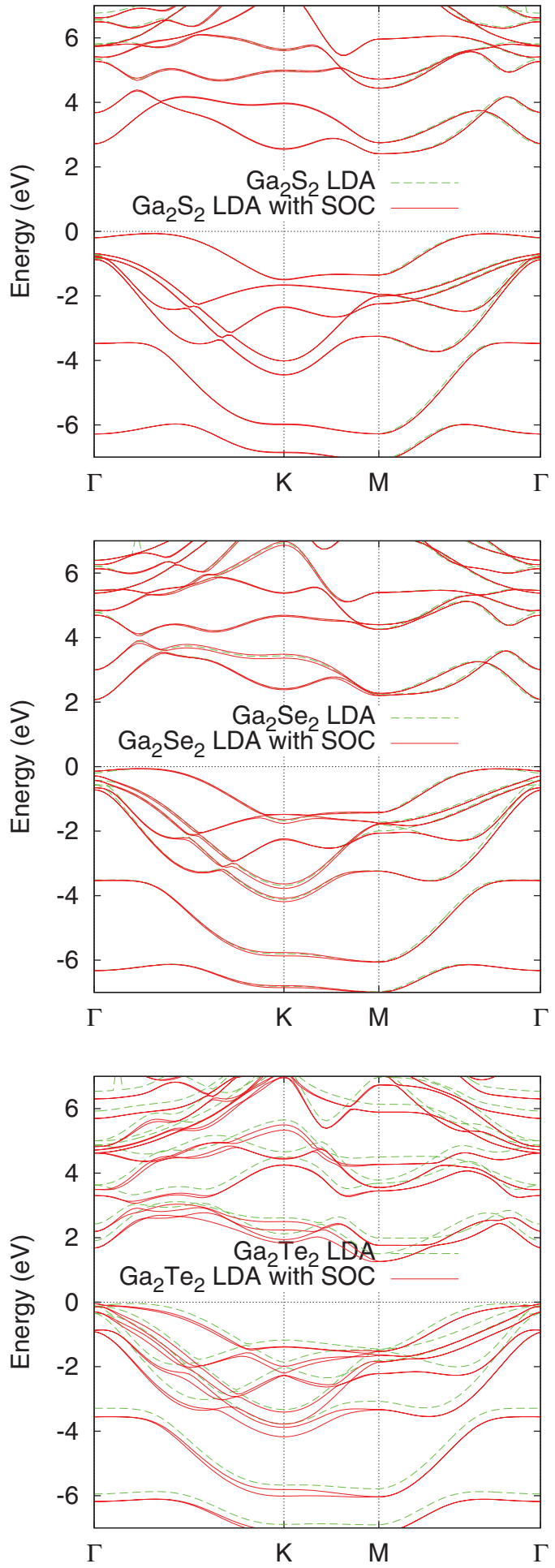

FIG. 5. (Color online) Comparison between the LDA band structures of $\mathrm{Ga}_{2} X_{2}$ with and without spin-orbit coupling (SOC).

between a filled and an empty band matches the energy of the main peak). In that range, the absorption coefficients of $\mathrm{Ga}_{2} X_{2}$ are comparable to and even exceed that of monolayer and bilayer graphene; therefore we suggest that ultrathin films of $\mathrm{Ga} X$ biased in vertical tunneling transistors with graphene electrodes could be used as an active element for detection of ultraviolet photons.

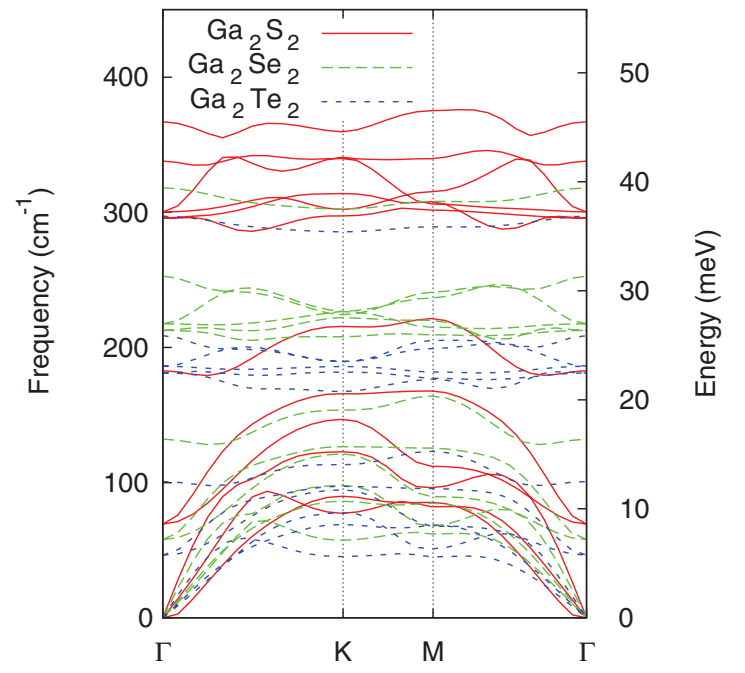

FIG. 6. (Color online) LDA phonon dispersion curves for $\mathrm{Ga}_{2} \mathrm{~S}_{2}$, $\mathrm{Ga}_{2} \mathrm{Se}_{2}$, and $\mathrm{Ga}_{2} \mathrm{Te}_{2}$.

In the case of the semilocal DFT calculations we also took spin-orbit coupling into account using a relativistic DFT approach. ${ }^{17}$ As can be seen in Fig. 5 some of the bands exhibit spin splitting, including the highest valence $\left(\Delta E_{S O}^{v, K}\right)$ and lowest conduction $\left(\Delta E_{S O}^{c, K}\right)$ bands near the $K$ point, most significant in $\mathrm{Ga}_{2} \mathrm{Te}_{2}$ (see Table III). While we were unable to calculate the spin-orbit splittings in HSE06 due to limited computational resources, we expect that they will exhibit a similar magnitude to that found in the semilocal band structures.

We have calculated the phonon dispersions for $\mathrm{Ga}_{2} \mathrm{X}_{2}$ using the force-constant approach in up to $4 \times 4$ supercells. The results are depicted in Fig. 6, suggesting that isolated atomic crystals of gallium chalcogenides, $\mathrm{Ga}_{2} \mathrm{X}_{2}$, are dynamically stable.

\section{CONCLUSION}

We have shown using first-principles density-functional theory that two-dimensional $\mathrm{Ga}_{2} X_{2}(X=\mathrm{S}, \mathrm{Se}$, and Te $)$ crystals are stable indirect-band-gap semiconductors with an unusual inverted sombrero valence band. The presence of saddle points along the $\Gamma-M$ line leads to a Lifshitz transition in the event of hole doping for which we have calculated the critical carrier density. We have provided an analytical fit of the valence-band edge and given a qualitative description of the optical absorption spectra, which suggest that ultrathin films of $\mathrm{Ga} X$ biased in vertical tunneling transistors with graphene electrodes could be used as an active element for detection of ultraviolet photons.

\section{ACKNOWLEDGMENTS}

We acknowledge financial support from the EPSRC Science and Innovation Award, the ERC Advanced Grant "Graphene and Beyond," the Royal Society Wolfson Merit Award, the Marie Curie project CARBOTRON, and the EC STREP "ConceptGraphene." 
${ }^{1}$ K. S. Novoselov, A. K. Geim, S. V. Morozov, D. Jiang, Y. Zhang, S. V. Dubonos, I. V. Grigorieva, and A. A. Firsov, Science 306, 666 (2004).

${ }^{2}$ A. K. Geim and K. S. Novoselov, Nat. Mater. 6, 183 (2007).

${ }^{3}$ Y. Kubota, K. Watanabe, O. Tsuda, and T. Taniguchi, Science 317, 932 (2007).

${ }^{4}$ M. P. Levendorf, C.-J. Kim, L. Brown, P. Y. Huang, R. W. Havener, D. A. Muller, and J. Park, Nature (London) 488, 627 (2012).

${ }^{5}$ K. F. Mak, C. Lee, J. Hone, J. Shan, and T. F. Heinz, Phys. Rev. Lett. 105, 136805 (2010).

${ }^{6}$ B. Radisavljevic, A. Radenovic, J. Brivio, V. Giacometti, and A. Kis, Nat. Nanotechnol. 6, 147 (2011).

${ }^{7}$ B. Radisavljevic, M. B. Whitwick, and A. Kis, ACS Nano 5, 9934 (2011).

${ }^{8}$ T. Georgiou, R. Jalil, B. D. Belle, L. Britnell, R. V. Gorbachev, S. V. Morozov, Y.-J. Kim, A. Gholinia, S. J. Haigh, O. Makarovsky,

L. Eaves, L. A. Ponomarenko, A. K. Geim, K. S. Novoselov, and A. Mishchenko, Nat. Nanotechnol. 8, 100 (2013).

${ }^{9}$ J. N. Coleman, M. Lotya, A. ONeill, S. D. Bergin, P. J. King, U. Khan, K. Young, A. Gaucher, S. De, R. J. Smith, I. V. Shvets, S. K. Arora, G. Stanton, H.-Y. Kim, K. Lee, G. T. Kim, G. S. Duesberg, T. Hallam, J. J. Boland, J. J. Wang, J. F. Donegan, J. C. Grunlan, G. Moriarty, A. Shmeliov, R. J. Nicholls, J. M. Perkins, E. M. Grieveson, K. Theuwissen, D. W. McComb, P. D. Nellist, and V. Nicolosi, Science 331, 568 (2011).

${ }^{10}$ D. Braga, L. I. Gutirrez, H. Berger, and A. F. Morpurgo, Nano Lett. 12, 5218 (2012).

${ }^{11}$ C. Ataca, H. Sahin, and S. Ciraci, J. Phys. Chem. C 116, 8983 (2012).
${ }^{12}$ C. H. Ho and S. L. Lin, J. Appl. Phys. 100, 083508 (2006).

${ }^{13}$ G. C. Bhar, S. Das, and K. L. Vodopyanov, Appl. Phys. B 61, 187 (1995).

${ }^{14}$ W. I. Milne and J. C. Anderson, J. Phys. D: Appl. Phys. 6, 2115 (1973).

${ }^{15}$ I. M. Lifshitz, Sov. Phys. JETP 11, 1130 (1960).

${ }^{16}$ S. J. Clark, M. D. Segall, C. J. Pickard, P. J. Hasnip, M. I. J. Probert, K. Refson, and M. C. Payne, Z. Kristallogr. 220, 567 (2005).

${ }^{17}$ G. Kresse and J. Furthmüller, Phys. Rev. B 54, 11169 (1996).

${ }^{18}$ J. P. Perdew, K. Burke, and M. Ernzerhof, Phys. Rev. Lett. 77, 3865 (1996).

${ }^{19}$ J. Heyd, G. E. Scuseria, and M. Ernzerhof, J. Chem. Phys. 118, 8207 (2003); A. V. Krukau, O. A. Vydrov, A. F. Izmaylov, and G. E. Scuseria, ibid. 125, 224106 (2006).

${ }^{20}$ K. Refson, P. R. Tulip, and S. J. Clark, Phys. Rev. B 73, 155114 (2006).

${ }^{21}$ F. Favot and A. Dal Corso, Phys. Rev. B 60, 11427 (1999).

${ }^{22}$ S. Park, B. Lee, S. H. Jeon, and S. Han, Curr. Appl. Phys. 11, S337 (2011).

${ }^{23}$ J. K. Ellis, M. J. Lucero, and G. E. Scuseria, Appl. Phys. Lett. 99, 261908 (2011)

${ }^{24}$ D. V. Rybkovskiy, I. V. Vorobyev, A. V. Osadchy, and E. D. Obraztsova, J. Nanoelectron. Optoelectron. 7, 65 (2012).

${ }^{25}$ Th. Köhler, Th. Frauenheim, Z. Hajnal, and G. Seifert, Phys. Rev. B 69, 193403 (2004).

${ }^{26}$ G. Onida, L. Reining, and A. Rubio, Rev. Mod. Phys. 74, 601 (2002). 